\title{
ANALYTICAL SOLUTIONS OF THE MODIFIED COULOMB POTENTIAL USING THE FACTORIZATION METHOD.
}

\author{
Akaninyene D. Antia ${ }^{1}$, Eno E. Ituen, Hillary P. Obong ${ }^{2}$, and Cecilia N. Isonguyo ${ }^{1}$ \\ ${ }^{1}$ Theoretical Physics Group,Department of Physics, University of Uyo, Nigeria. \\ ${ }^{2}$ Theoretical Physics Group, Department of Physics, University of Port Harcourt, Choba, \\ P. M. B. 5323, Port Harcourt, Nigeria.
}

\begin{abstract}
We have solved exactly Schrödinger equation with modified Coulomb Potential under the framework of factorization method. Energy levels and the corresponding wave functions in terms of associated Laquerre function are also obtained. For further guide to interested readers we have computed the energy eigenvalue for some selected elements for various values of $n$ and $l$.
\end{abstract}

\section{KEYWORDS}

Modified coulomb potential, Schrodinger equation, bound state solution, factorization method.

\section{INTRODUCTION}

The exact bound-state solutions of the Schrödinger equation with physically significant potentials play a major role in quantum mechanics. And one of the important tasks in theoretical physics is to obtain exact solution of the Schrödinger equation for special potential. In recent years, exact and approximate solutions of Schrödinger equation with different potentials have attracted much interest [1-12].

The exact solutions of the Schrödinger equation are only possible for some potentials of physical interest $[7,13,14]$. It is well known that these exact solutions of the wave equations are only possible in cases such as harmonic oscillator, pseudoharmonic and Mie-type potentials $[5,15]$. However, for an arbitrary $l$-state, many potential of the quantum system could only be solved by approximation method $[16,17]$.

Different methods have been developed in obtaining the exact or approximate solutions of Schrödinger, Klein-Gordon and Dirac equations for any potential of interest. Among such methods include the shape invariant method [18], supersymmetric quantum mechanics approach

(SUSYQM) [19], Nikiforov-Uvarov (NU) [20], asymptotic iteration method (AIM) [21], $\frac{1}{\mathrm{~N}}$ expansion method [22], factorization method [23] and others [24].

The relativistic Coulomb and oscillator potential problems including their bound-state specta and wave functions have already been established for a long time [25], and ref. therein and their nonrelativistic limits reproduce the usual Schrödinger Coulomb and Schrödinger oscillator solutions respectively. Chen and Dong [26] obtained the exact solution of the Schrödinger equation for the 
Coulomb potential plus ring-shaped potential which has possible applications to ring-shaped organic molecules like cyclic polyenes and benzene.

In this paper we consider the modified Coulomb potential defined as

$$
V(r)=I-\frac{Z e^{2}}{r},
$$

where $I$ is the threshold potential, $Z$ is the atomic number of the atom, $e$ is the charge of electron. The effective potential $V_{\text {eff }}(r)$ of Eq. (1) is given as

$$
V_{e f f}(r)=I-\frac{Z e^{2}}{r}+\frac{l(l+1) \hbar^{2}}{2 \mu r^{2}}
$$

This potential has great applications in many branches of physics and chemistry such as nuclear, atomic and molecular physics, nuclear chemistry and other related areas. The aim of this paper is to solve the Schrödinger equation under modified Coulomb potential within the framework of factorization method [27].

\section{FACTORIZATION METHOD}

In the spherical coordinates, the Schrödinger wave equation is

$$
\begin{aligned}
& \frac{-\hbar^{2}}{2 \mu}\left[\frac{1}{r^{2}} \frac{\partial}{\partial r}\left(r^{2} \frac{\partial}{\partial r}\right)+\frac{1}{r^{2} \sin \theta} \frac{\partial}{\partial \theta}\left(\sin \theta \frac{\partial}{\partial \theta}\right)+\frac{1}{r^{2} \sin ^{2} \theta} \frac{\partial^{2}}{\partial \varphi^{2}}\right] \Psi(r, \theta, \varphi) \\
& +V_{e f f}(r) \Psi(r, \theta, \varphi)=E \Psi(r, \theta, \varphi),
\end{aligned}
$$

where $V_{\text {eff }}(r)$ in this case is the effective potential of the modified Coulomb potential of Eq. (2). In order to find exact solution of Eq. (3), we give spherical total wave function as

$$
\Psi(r, \theta, \varphi)=R(r) \Theta(\theta) \Phi(\varphi)
$$

Substituting Eq. (4) into Eq. (3) yields the wave equation for the effective potential separated into independent variable as the following equations:

$$
\begin{aligned}
& \frac{d^{2} R(r)}{d r^{2}}+\frac{2}{r} \frac{d R(r)}{d r}+\frac{2 \mu}{\hbar^{2}}\left[E-V_{\text {eff }}(r)\right] R(r)=0, \\
& \frac{d^{2} \Phi(\theta)}{d \theta^{2}}+\cot \frac{d \Theta(\theta)}{d \theta}+\left[\lambda-\frac{m^{2}}{\sin ^{2} \theta}\right] \Theta(\theta)=0, \\
& \frac{d^{2} \Phi(\varphi)}{d \varphi^{2}}+m^{2} \Phi(\varphi)=0
\end{aligned}
$$

where $\lambda=l(l+1)$ and $m^{2}$ are separation constants. Equation (6) and (7) are spherical harmonic $Y_{l m}(\theta, \varphi)=\Theta(\theta) \Phi(\varphi)$ whose solution is well known [28]. Therefore, our interest is on Eq. (5). 


\section{THE SOLUTIONS OF THE RADIAL PART OF THE SCHRÖDINGER WITH MODIFIED COULOMB POTENTIAL}

Substituting Eq. (2) into Eq. (5), we can rewrite the radial part of the Schrödinger equation with the effective potential as

$$
\frac{d^{2} R(r)}{d r^{2}}+\frac{2}{r} \frac{d R(r)}{d r}+\frac{2 \mu}{\hbar^{2}}\left[E-I+\frac{Z e^{2}}{r}-\frac{\lambda \hbar^{2}}{\mu r^{2}}\right] R(r)=0
$$

By a change of variable of the form

$$
\rho=\alpha r,
$$

Eq. (8) is written as

$$
\rho R^{\prime \prime}(\rho)+2 R^{\prime}(\rho)+\left[\frac{2 \mu}{\hbar^{2} \alpha^{2}}(E-I) \rho+\frac{2 \mu Z e^{2}}{\hbar^{2} \alpha}-\frac{\lambda}{\rho}\right] R(\rho)=0
$$

Writing ansatz for the function in eq. (10) as

$$
R(\rho)=U(\rho) L_{n, m}^{(\alpha, \beta)}(\rho)
$$

and substituting Eq. (11) into Eq. (10), and after a little algebraic, we get

$$
\rho L^{\prime \prime}(\rho)+\left(\frac{2 \rho U^{\prime}(\rho)}{U(\rho)}+2\right) L^{\prime}(\rho)+\left(\frac{\rho U^{\prime \prime}(\rho)}{U(\rho)}+\frac{2 U^{\prime}(\rho)}{U(\rho)}+\frac{2 \mu}{\hbar^{2} \alpha^{2}}\left(E_{-} I\right) \rho+\frac{2 \mu Z e^{2}}{\hbar^{2} \alpha}-\frac{\lambda}{\rho}\right) L(\rho)=0 .
$$

12

To obtain the wave function $U(\rho)$ we compare Eq. (12) with the following associated Laquerre deferential equation [27] and ref there in.

$$
\rho L_{n, m}^{\prime \prime(\alpha, \beta)}(\rho)+(1+\alpha-\beta \rho) L_{n, m}^{\prime(\alpha, \beta)}(\rho)+\left[\left(n-\frac{m}{2}\right) \beta-\frac{m}{2}\left(\alpha+\frac{m}{2}\right) \frac{1}{\rho}\right] L_{n, m}^{(\alpha, \beta)}(\rho)=0
$$

So we obtain the $U(\rho)$ as

$$
U(\rho)=e^{\frac{-\beta \rho}{2}} \rho^{\frac{(\alpha-1)}{2}}
$$

Substituting Eq. (14) into Eq. (11) yields the wave function for this system as

$$
R(\rho)=e^{\frac{-\beta \rho}{2}} \rho^{\frac{(\alpha-1)}{2}} L_{n, m}^{(\alpha, \beta)}(\rho)
$$

where $L_{n, m}^{(\alpha, \beta)}(\rho)$ is the associated Laquerre polynomial.

Here, we note that the solution of associated Laquerre in the Rodriques representation are:

$$
L_{n, m}^{(\alpha, \beta)}(x)=\frac{A_{n, m}(\alpha, \beta)}{(\rho)^{\alpha+\frac{m}{2}} e^{-\beta \alpha r}}\left(\frac{d}{d x}\right)^{n-m}\left(\rho^{n+\alpha} e^{-\beta \alpha r}\right),
$$

where $A_{n, m}(\alpha, \beta)$ is the normalization constants which is also obtained as

$$
A_{n, m}(\alpha, \beta)=(-1)^{m} \sqrt{\frac{\beta^{\alpha+m+1}}{\Gamma(n-m+1) \Gamma(n+\alpha+1)}}
$$

To obtain the energy eigenvalue we substitute Eq. (14) into Eq. (12) and carry out the required derivative to have: 


$$
\begin{aligned}
& \rho L^{\prime \prime}(\rho)+(1+\alpha-\beta \rho) L^{\prime}(\rho)+ \\
& {\left[\left(\frac{\beta^{2}}{4}+\frac{2 \mu}{\hbar^{2} \alpha^{2}}(E-I)\right) \rho-\frac{(\alpha+1)}{2} \beta+\frac{2 \mu Z e^{2}}{\hbar^{2} \alpha}+\left(\frac{1}{4}(\alpha-1)(\alpha-3)+(\alpha-1)-\lambda\right) \frac{1}{\rho}\right] L(\rho)=0}
\end{aligned}
$$

18

Comparing Eq. (18) with Eq. (13), we have

$$
\begin{gathered}
\beta=\frac{4 \mu Z e^{2}}{\hbar^{2} \alpha(2 n-m+\alpha+1)}, \\
m=-\alpha \pm \sqrt{4 \lambda+1}, \text { where } \lambda=l(l+1), \\
E=I-\frac{16 \mu Z^{2} e^{4}}{8 \hbar^{2}(2 n-m+\alpha+2)^{2}}
\end{gathered}
$$

Where $\alpha, \beta, m$ are polynomial parameters. $\alpha$ and $\beta$ are the arbitrary numbers, $n$ is the quantum number and $m$ is the magnetic quantum number.

Equation (21) is the energy eigenvalue of the modified coulomb potential. Using the magnetic quantum number $m$ the energy eigenvalue of Eq. (21) becomes

$$
E=I-\frac{16 \mu Z^{2} e^{4}}{8 \hbar^{2}(2 n+2 \alpha+1-\sqrt{4 \lambda+1})^{2}}
$$

But for ordinary Laquerre polynomial, $\alpha=0$ thus Eq. (22) becomes

$$
E_{n, l}=I-\frac{16 \mu Z^{2} e^{4}}{8 \hbar^{2}(2 n+1-\sqrt{4 l(l+1)+1})^{2}}
$$

If $I=0$ and we set $\mu \rightarrow \frac{\mu}{4}$ for $l=0$, Eq. (23) reduces to the Coulomb energy of the form

$$
E_{n}^{C}=-\frac{\mu Z^{2} e^{4}}{8 \hbar^{2} n^{2}}
$$

The energy states eigenvalue of Eq. (24) for the five(5) selected elements Hydrogen (H), Lithium (Li), Sodium (Na), Potassium $(\mathrm{K})$ and Copper $(\mathrm{Cu})$ have been calculated. The threshold potential $I(\mathrm{eV})$ of these elements is presented in Table 1. The numerical behaviours of the energy of the selected elements with various values of $n$ and $l$ for $\mu=\hbar=1$ and $e=1.6 \times 10^{-19} C$ are presented in Tables $2-6$. From the computed results it can be observed that the degeneracies exist as expected in tables $2-6$.

Table 1: Threshold potential I for some selected elements

\begin{tabular}{|l|l|}
\hline Element & \multicolumn{1}{|c|}{ I $($ eV $)$} \\
\hline Hydrogen $\mathrm{H}$ & 13.6 \\
\hline Lithium $\mathrm{Li}$ & 5.39 \\
\hline Sodium $\mathrm{Na}$ & 5.14 \\
\hline Potassium $\mathrm{K}$ & 4.74 \\
\hline Copper $\mathrm{Cu}$ & 7.73 \\
\hline
\end{tabular}


International Journal of Recent advances in Physics (IJRAP) Vol.4, No.1, February 2015

Table 2: Energy eigenvalue for Hydrogen, $\mathrm{H}$

\begin{tabular}{|ll|l|l|l|}
\hline & \multicolumn{3}{|c|}{ Energy eigenvalue $E_{n, l}(\mathrm{eV}) \times 10^{-8}$} \\
\hline & \multicolumn{1}{c|}{$l=0$} & \multicolumn{1}{c|}{$l=1$} & $l=2$ \\
\hline 1 & & -231.1 & -102.5 & -57.8 \\
\hline 2 & -102.5 & -57.8 & -37.0 \\
\hline 3 & & -57.8 & -37.0 & -25.7 \\
\hline 4 & -37.0 & -25.7 & -18.9 \\
\hline 5 & & -25.7 & -18.9 & -14.4 \\
\hline
\end{tabular}

Table 3: Energy eigenvalue for Lithium, Li

\begin{tabular}{|ll|l|l|l|}
\hline & \multicolumn{4}{|c|}{ Energy eigenvalue $E_{n, l}(\mathrm{eV}) \times 10^{-8}$} \\
\hline & $n$ & \multicolumn{1}{c|}{$l=0$} & \multicolumn{1}{c|}{$l=1$} & \multicolumn{1}{c|}{$l=2$} \\
\hline 1 & & -6238.5 & -2772.7 & -1559.6 \\
\hline 2 & & -2772.7 & -1559.6 & -998.2 \\
\hline 3 & -1559.6 & -998.2 & -693.2 \\
\hline 4 & & -998.2 & -693.2 & -509.3 \\
\hline 5 & & -693.2 & -509.3 & -389.9 \\
\hline
\end{tabular}

Table 4: Energy eigenvalue for Sodium $(\mathrm{Na})$

\begin{tabular}{|ll|l|l|l|}
\hline & \multicolumn{4}{|l|}{ Energy eigenvalue $E_{n, l}(\mathrm{eV}) \times 10^{10}$} \\
\hline & \multicolumn{1}{|c|}{$l=0$} & \multicolumn{1}{l|}{$l=1$} & $l=2$ \\
\hline 1 & & -3075.3 & -1366.8 & -768.8 \\
\hline 2 & -1366.8 & -768.8 & -492.1 \\
\hline 3 & & -768.8 & -492.1 & -341.7 \\
\hline 4 & & -492.1 & -341.7 & -251.0 \\
\hline 5 & & -341.7 & -251.0 & -192.2 \\
\hline
\end{tabular}

Table 5: Energy eigenvalue for Potassium (K)

\begin{tabular}{|ll|l|l|l|}
\hline & & \multicolumn{3}{|c|}{ Energy eigenvalue $E_{n, l}(\mathrm{eV}) \times 10^{-11}$} \\
\hline & \multicolumn{1}{|c|}{$l=0$} & \multicolumn{1}{l|}{$l=1$} & \multicolumn{1}{l|}{$l=2$} \\
\hline 1 & & -1584.8 & -704.4 & -396.2 \\
\hline 2 & & -704.4 & -396.2 & -253.6 \\
\hline 3 & & -396.2 & -253.6 & -176.1 \\
\hline 4 & & -253.6 & -176.1 & -129.4 \\
\hline 5 & & -176.1 & -129.4 & -99.1 \\
\hline
\end{tabular}


International Journal of Recent advances in Physics (IJRAP) Vol.4, No.1, February 2015

Table 6: Energy eigenvalue for Copper $(\mathrm{Cu})$

\begin{tabular}{|ll|l|l|l|}
\hline & \multicolumn{4}{|c|}{ Energy eigenvalue $E_{n, l}(\mathrm{eV}) \times 10^{-11}$} \\
\hline & \multicolumn{1}{|c|}{$l=0$} & \multicolumn{1}{l|}{$l=1$} & $l=2$ \\
\hline 1 & & -5633.6 & -2503.8 & -1408.4 \\
\hline 2 & & -2503.8 & -1408.4 & -901.4 \\
\hline 3 & -1408.4 & -901.4 & -626.0 \\
\hline 4 & & -901.4 & -626.0 & -459.9 \\
\hline 5 & & -626.0 & -459.9 & -352.1 \\
\hline
\end{tabular}

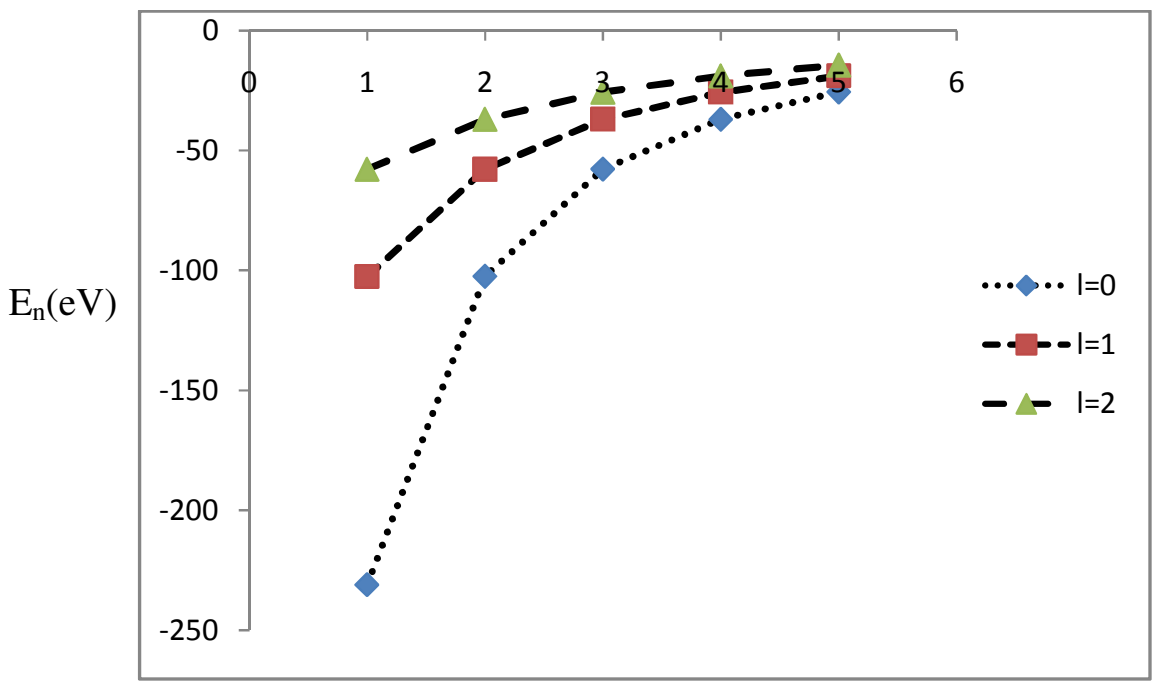

$n$

Fig. 1: Energy $E_{n}(e V)$ versus $n$ for Hydrogen $(H)$

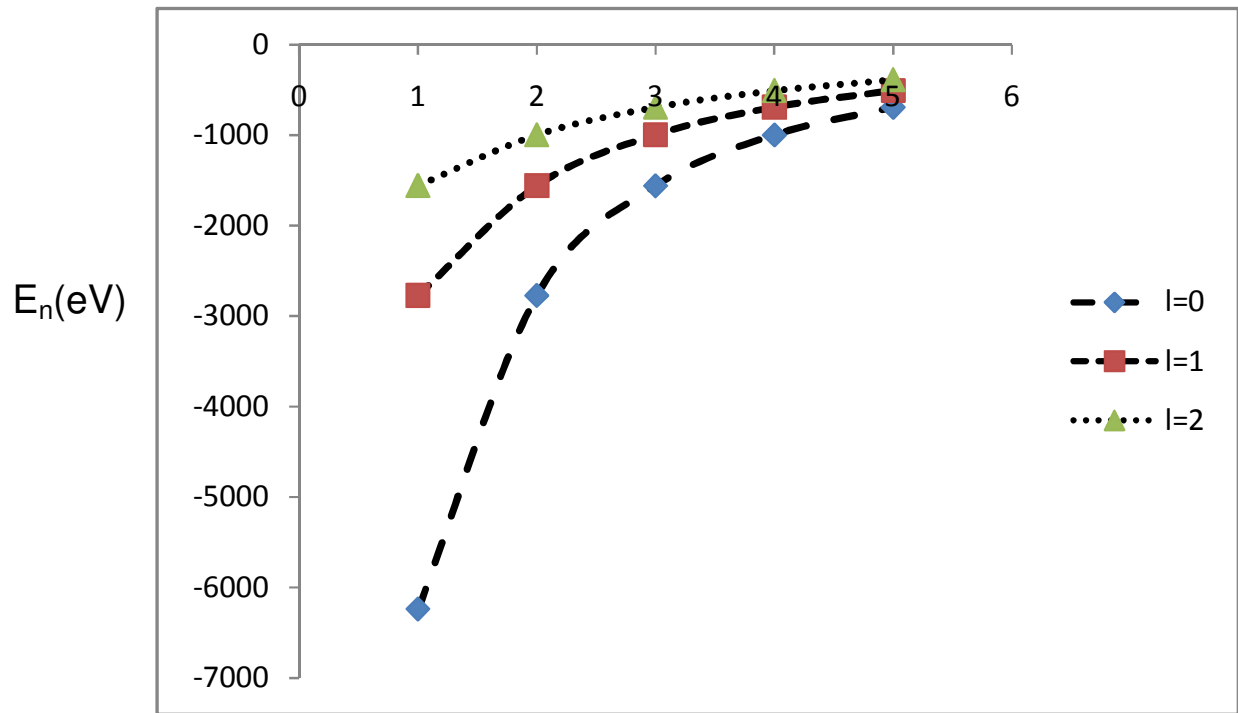

n

Fig. 2: Energy $\mathrm{E}_{\mathrm{n}}(\mathrm{eV})$ versus $\mathrm{n}$ for Lithium (Li) 
International Journal of Recent advances in Physics (IJRAP) Vol.4, No.1, February 2015

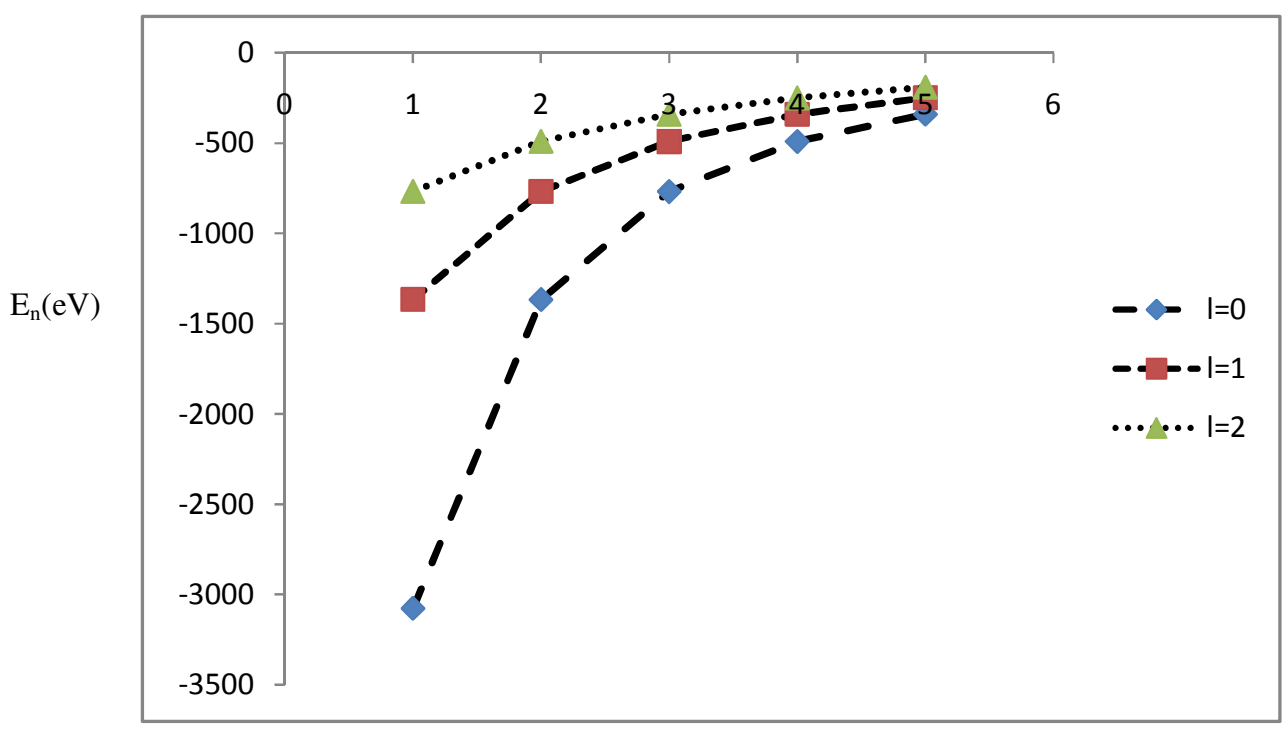

$\mathrm{n}$

Fig. 3: Energy $E_{n}(e V)$ versus $n$ for Sodium $(\mathrm{Na})$

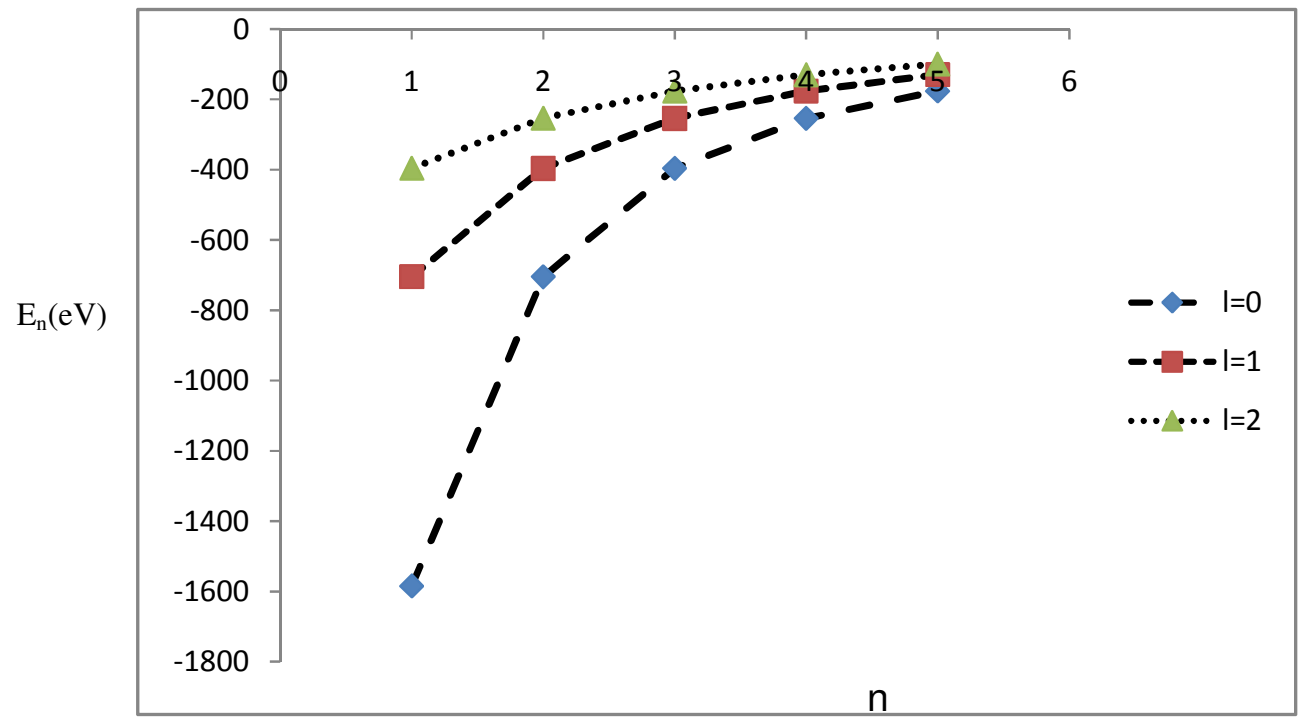

Fig. 4: Energy $\mathrm{E}_{\mathrm{n}}(\mathrm{eV})$ versus $\mathrm{n}$ for Potassium (K) 


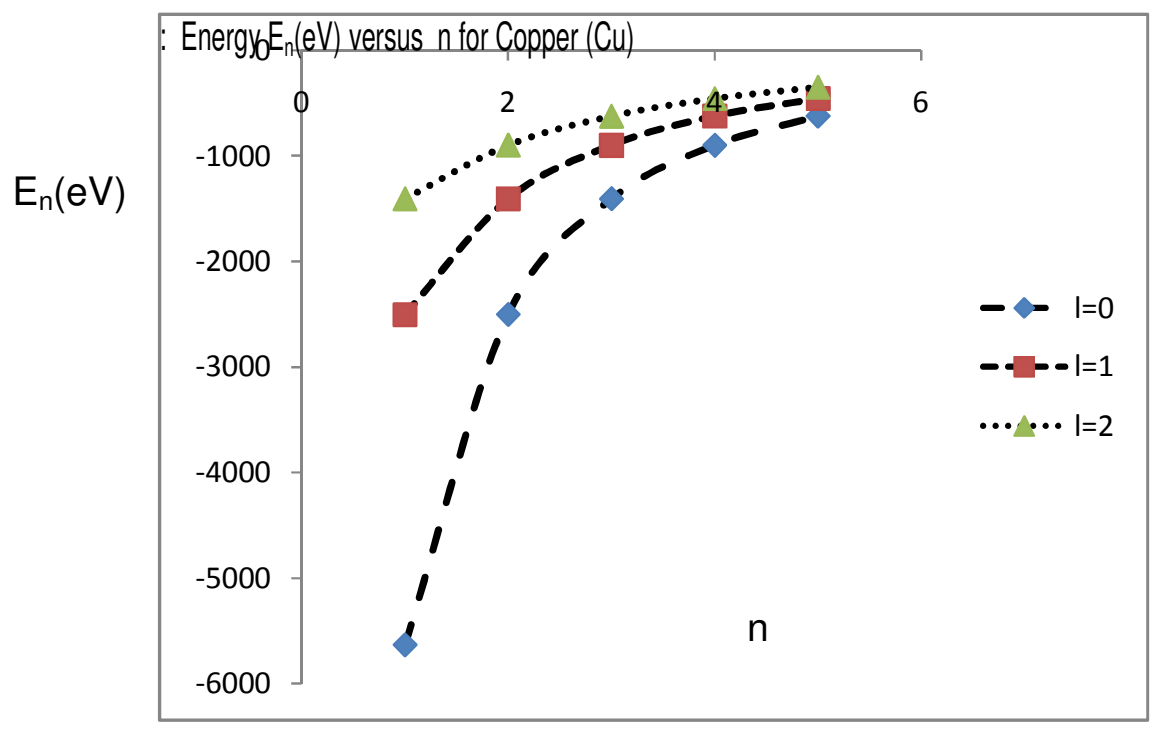

Fig 5: Energy En(eV) versus $n$ for Copper $(\mathrm{Cu})$

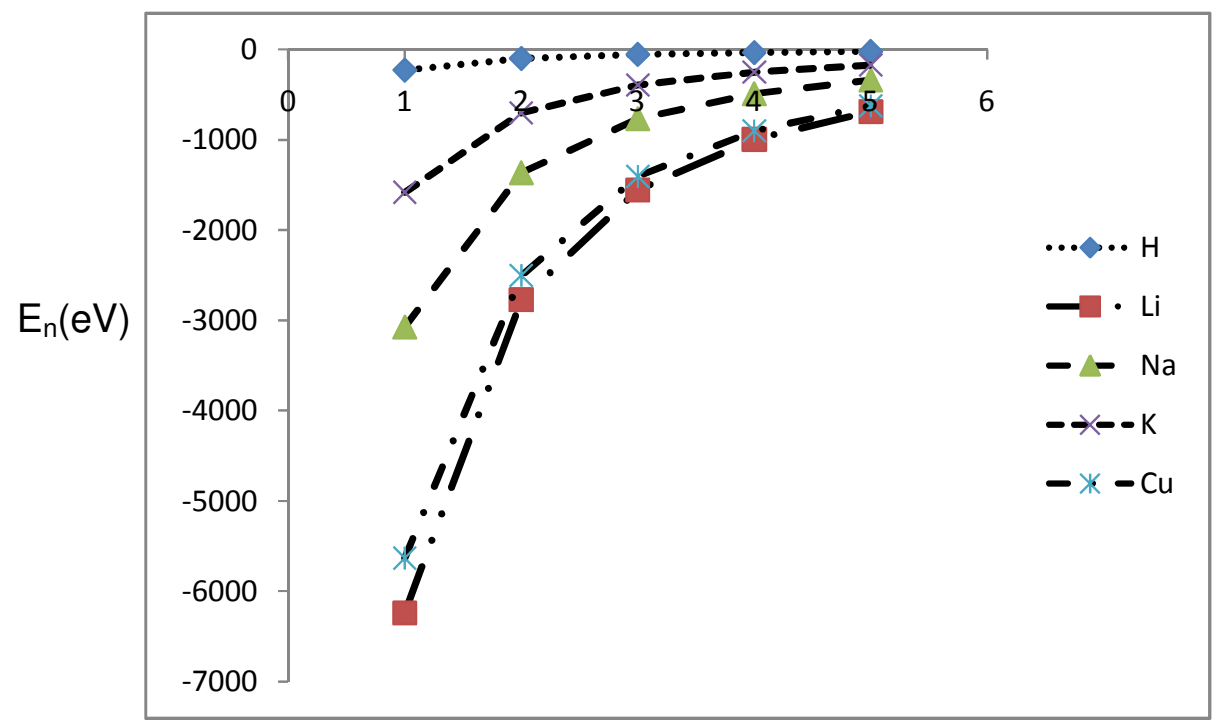

$\mathrm{n}$

Fig. 6: Energy $\mathrm{E}_{\mathrm{n}}(\mathrm{eV})$ comparison for selected elements for $\mathrm{l}=0$ 


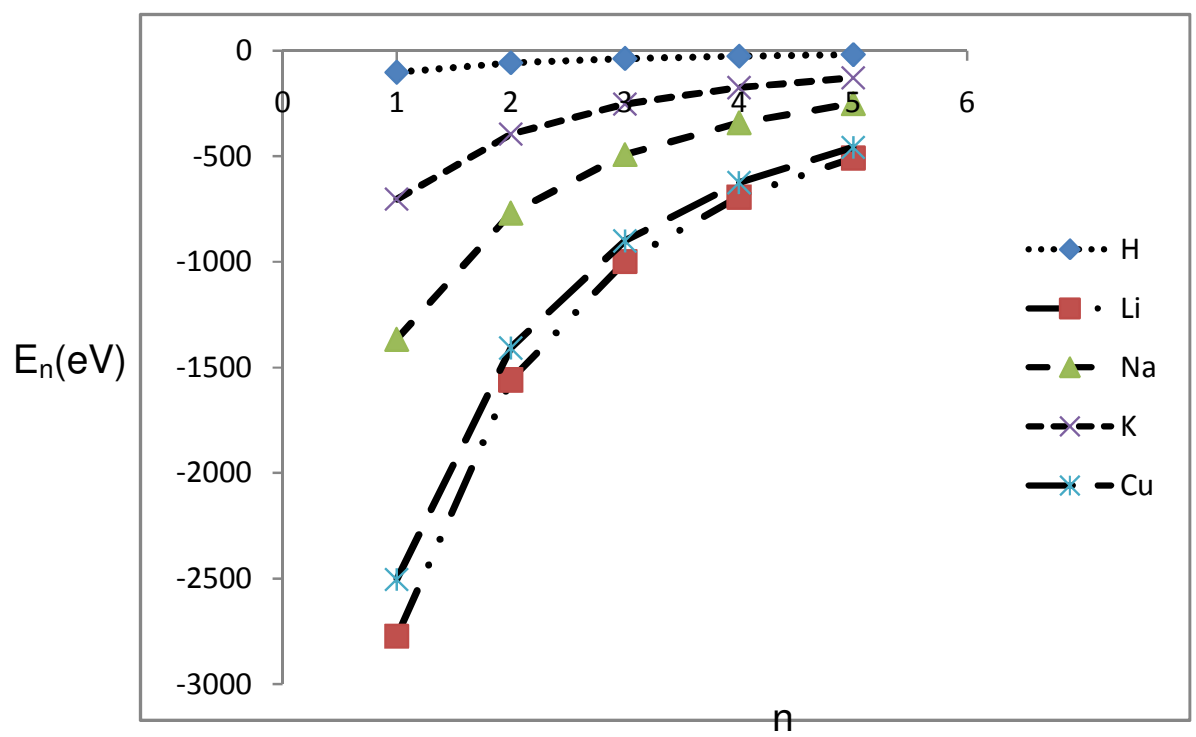

Fig. 7: Energy $E_{n}(e V)$ comparison for selected elements for $l=1$

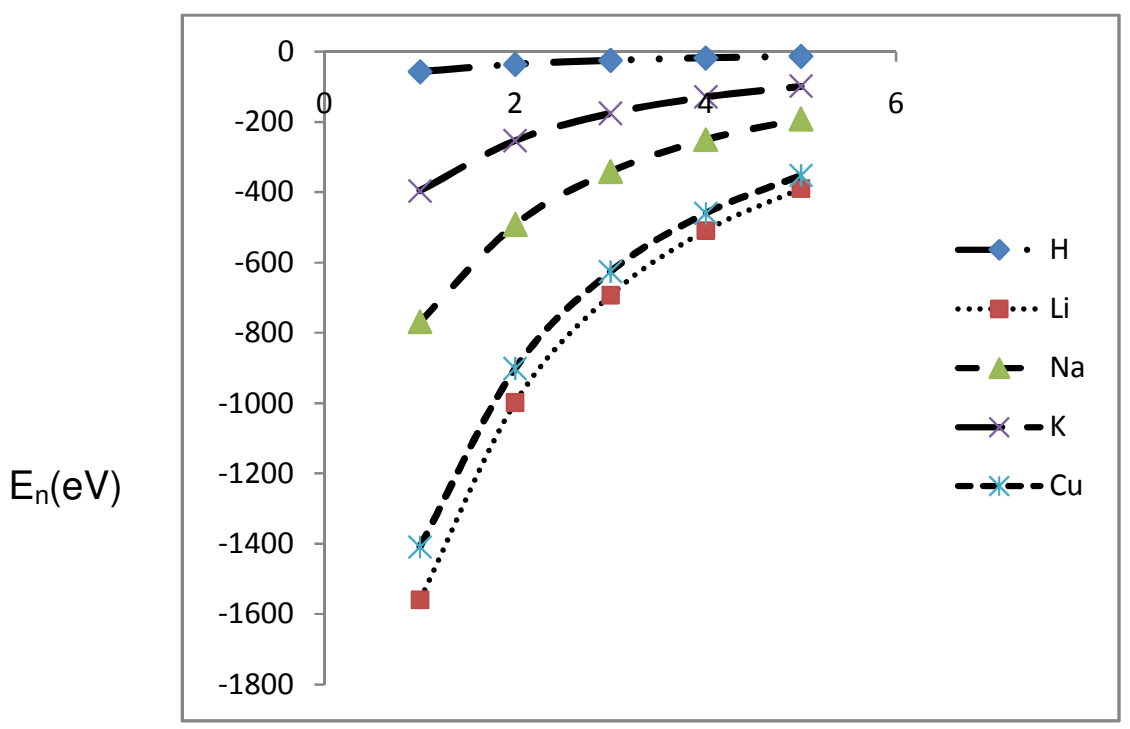

$n$

Fig. 8: Energy $E_{n}(e V)$ comparison for selected elements for $1=2$ 


\section{CONCLUSION}

In this paper, we have obtained the exact solutions of the Schrödinger equation for modified Coulomb potential using the factorization method. The energy eigenvalue and the wave function expressed in terms of associated Laquerre function are obtained. Numerical data for the energy spectrum are discussed for some selected elements like $\mathrm{H}, \mathrm{Li}, \mathrm{Na}, \mathrm{K}$ and $\mathrm{Cu}$ indicating usefulness for other physical systems.

\section{REFERENCES}

1. Ikot, Akpan N., Awoga, Oladuyoye A., Hassanabdi, Hassan and Maghsoodi, Elham (2014). Analytical Approximate solution of Schrödinger equation in D dimensions with quadratic Exponential-Type potential for Arbitrary $l$ - state. Commun. Theor. Phys. 61, 457-463.

2. Aktas, M. and Sever, R. (2004). Exact supersymmetric solution of Schrödinger equation for central confining potentials by using NIkiforov-Uvarov method. Journal of Molecular structure - Theochem, 710, 223-228.

3. Hassanabadi, H., Zarriskamar, S. and Rayabi, A. A. (2011). Exact solutions of D-dimensional Schrödinger equation for energy-dependent potential by Nikiforov-Uvarov method. Communication in Theoretical Physics, 55, 541-544.

4. Ikhdair, S. M. and Sever, R. (2008). Exact solution of the Mie type potential in the N-dimension Schrödinger equation. Journal of Molecular structures: Theochem, 855, 13-27.

5. Agboola, D. (2011). Complete Analytical Solutions of the Mie-type potentials in N-dimensions. Acta Physics Polonica A, 120(3), 371-376.

6. Antia, D. A., Ikot, A. N. and Akpabio, L. E. (2010). Exact Solutions of the Schrödinger equation with Manning-Rosen potential plus a ring-shaped like potential by Nikiforov-Uvarov method. European Journal of Scientific Research, 46(1), 107-118.

7. Antia, A. D., Ikot, A. N., Ituen, E. E. and Akpbio, L. E. (2012). Analytical solution of Schrödinger equation with Eckart potential plus Hulthen potential via Nikiforov-Uvarov method. Palestine Journal of Mathematics, 1(2), 104-109.

8. Badalov, V. H., Ahmadov, H. I. and Badalov, S. V. (2009). Analytical solutions of the Schrödinger equation with the Woods-Saxon potential for arbitrary $l$-state. International Journal of Modern Physics E, 18, 631-641.

9. Ikot, A. N., Akpabio, L. E. and Umoren, E. B. (2011). Exact solution of Schrödinger equation with inverted Woods-Saxon and Manning-Rosen potential. Journal of Scientific Research, 3(1), 25-33.

10. Ikot, A. N. (2011). Analytical solutions of with generalized hyperbolic potential using NikiforovUvarov method. African Review Physics, 60026, 221-228.

11. Ikot, A. N. and Akpabio, I. O. (2012). Bound state solutions of the Schrödinger equation for a more general Woods-Saxon potential with arbitrary $l$-state. Chinese Physics Letters, 29(9), 090302.

12. Sever, R., Tezcan, C. Aktas, M. and Yesutas, O. (2007). Exact solutions of the Schrödinger equation for Pseudoharmonic potential. Journal of Mathematical Chemistry, 43, 845-854.

13. Awoga, O. A., Ikot, A. N., Akpan, I. O. and Antia, A. D. (2012). Solution of Schrödinger equation with exponential coshine-screened potential. Indian Journal of pure and Applied Physics, 50, 217-233.

14. Hsassnabadi, H. Zarriskamar, S. and Rajabi, A. A. (2011). Exact solution of D-dimensional Schrödinger equation for energy-dependent potential by Nikiforov-Uvarov method. Communciation in Theoretical Physics, 55, 541-544.

15. Ikhdair, S. M. and Sever, R. (2007). Exact polynomial eigen solutions of the Schrödinger equation for the pseudoharmonic potential. Journal of molecular structures - Theochem, 806, 103-113.

16. Ikot, A. N., Akpabio, L. E and Uwah, E. J. (2011). Bound state solutions of the Klein-Gordon equation with the Hulthen potential. Electronic Journal of Theoretical physics 8(25), 225-232.

17. Greene, R. L. and Aldrich, C. (1976). Variation wave functiond for a screened coulomb potential. Physcial Revision A, 14, 2363-2666.

18. Morales, D. A. (2004). Supersymmetric improvement of the Pekis approximation for the rotating more potential. Chemical physics Letter, 394, 68-75.

19. Cooper, F. Khare, A. and Sukhatme, U. P. (1995). Supersymmetry and quantum mechanics, Physics Reports, 251, 257-385. 
International Journal of Recent advances in Physics (IJRAP) Vol.4, No.1, February 2015

20. Nikiforov, A. F. and Uvarov, U. B. (1988). Special functions of mathematical physics. Basel: Birkhauser.

21. Ciftici, H, Hall, R. L. and Saad, N. (2003), asymptotic iteration method for Eigen value problems. Journal of physics A: mathematical and General, 36 (47), 11807 - 11816.

22. Bag, M. , Panja, M. M., Dutt, R. and Varshni, Y. P. (1992)

23. S. Ikhdair and R. Sever (2008) Exact polynomial eigen solution of Schrodinger for pseudo harmonies potential. international Journal of Modern physics 19, 221-229

24. Dong, S., Gercia - Ravalo, J. and Dong, S. H. (2007) Quantization rule solution to the Hulthen potential in arbitrary l-state. Physica scripta, 76, $393-401$.

25. Pahlavani, M., R. , Sadeghi, J. and Ghezelbash, M. (2009). Solution of the central Woods-Saxon potential in $l \neq 0$ case using mathematical modification method. Applied sciences 11, $106-113$.

26. Chen, Y. C. , Dong, S. H. (2005). Physics. Lett . A 335, 374.

27. Sadeghi, J. and Pourhassan B. (2008). Exact solution of the Non-central modified kratzer potential by the factorization method. Electronic Journal of theoretical Physics 5(17), 193 - 202. 